\title{
Crenças em dor crônica: validação do Inventário de Atitudes frente à Dor para a língua portuguesa
}

\author{
CHRONIC PAIN BELIEFS: VALIDATION OF THE SURVEY OF PAIN ATTITUDES \\ FOR THE PORTUGUESE LANGUAGE
}
CREENCIAS EN DOLOR CRÓNICO: VALIDACIÓN DEL INVENTARIO DE ACTITUDES FRENTE AL DOLOR PARA LA LENGUA PORTUGUESA.

Cibele Andrucioli de Mattos Pimenta', Diná de Almeida Lopes Monteiro da Cruz ${ }^{2}$

\section{RESUMO}

Este estudo validou o Inventário de Atitudes frente à Dor - versão reduzida (IAD-breve) para a língua portuguesa. Sessenta e nove pacientes brasileiros foram avaliados. Os pacientes foram mulheres $(71 \%)$, a idade média foi 50,8 anos $( \pm 15,4)$, a escolaridade média 7,4 anos $( \pm 4,8)$, e as dores mais freqüentes foram de etiologia musculoesquelética $(39,1 \%)$, relacionadas ao câncer $(34,8 \%)$ e neuropáticas $(20,3 \%)$. A análise fatorial resultou em sete domínios (Controle, Dano-físico, Incapacidade, Cura Médica, Emoção, Medicação e Solicitude), como no instrumento original. A consistência interna, avaliada pelo $\propto$ Chronbach, variou de 0,55 a 0,89, valores considerados moderados e bons. A versão em português apresentou equivalência conceitual à versão em inglês. A disponibilidade do IAD-breve, em português, pode contribuir para a melhoria da pesquisa e prática clínica em pacientes com dor crônica.

\section{DESCRITORES}

Dor.

Atitude.

Cognição.

Medição da dor.

\section{ABSTRACT}

This study validated the Survey of Pain Attitudes - brief version (SOPA) for the Portuguese language. Sixty-nine Brazilian patients were evaluated. Patients were female $(71 \%)$, the mean age was 50.8 years $( \pm 15.4)$, the mean schooling was 7.4 years $( \pm 4.8)$, and the most frequent pain etiologies were: musculoskeletal (39.1\%), cancer-related $(34.8 \%)$, and neuropathic $(20.3 \%)$. Factorial analysis produced seven domains (Control, Harm, Disability, Medical Cure, Emotion, Medication, Solicitude), as in the original instrument. Indices of reliability tests ( $\mu$ Chronbach) ranged from .55 to .89 , values that are accepted as moderate and good. The Portuguese language version showed conceptual equivalence to the English language version. The availability of SOPA-brief version in Portuguese may contribute for the improvement of research and clinical practices on chronic pain.

KEY WORDS
Pain.
Attitude.
Cognition.
Pain measurement.

\section{RESUMEN}

En este estudio se validó el Inventario de Actitudes frente al Dolor - versión reducida (IAD-breve) para el idioma portugués. Fueron evaluados sesenta y nueve pacientes brasileños. Los pacientes fueron mujeres $(71 \%)$, la edad media fue de 50,8 años $( \pm 15,4)$, la escolaridad media 7,4 años $( \pm 4,8)$, y los dolores más frecuentes fueron de etiología músculo esquelética $(39,1 \%)$, relacionadas al cáncer $(34,8 \%)$ y neuropáticas $(20,3 \%)$. El análisis factorial resultó en siete dominios (Control, Daño físico, Incapacidad, Cura Médica, Emoción, Medicación y Solicitud), como en el instrumento original. La consistencia interna, evaluada por el $\mu$ Chronbach, varió de 0,55 a 0,89 , valores considerados moderados y buenos. La versión en portugués presentó equivalencia conceptual a la versión en inglés. La disponibilidad del IADbreve, en portugués, puede contribuir en la mejoría de la investigación y la práctica clínica en pacientes con dolor crónico.

\section{DESCRIPTORES \\ Dolor. \\ Actitude. \\ Cognición. \\ Dimensión del dolor.}

1 Enfermeira. Professora Livre-Docente do Departamento de Enfermagem MédicoCirúrgica da Escola de Enfermagem da Universidade de São Paulo (EEUSP). parpca@usp.br

2 Enfermeira. Professora Livre-Docente do Departamento de Enfermagem MédicoCirúrgica da EEUSP. mtmllf@usp.br 


\section{INTRODUÇÃO}

A apreciação e expressão da dor crônica resultam de interações entre o impulso nóxico, elementos biológicos, emocionais e cognitivos do indivíduo ${ }^{(1)}$. Estudos têm apontado que crenças disfuncionais são capazes de exacerbar a experiência de dor, acarretar piora na funcionalidade física e psíquica e prejudicar a adesão ao tratamento, sendo freqüentes em doentes com dor crônica $^{(2)}$.

Em 1976 introduziram-se os preceitos da Teoria Cognitiva Comportamental na área de controle da dor crônica ${ }^{(3-4)}$. Os valores, as atitudes, as crenças, as expectativas, o julgamento do doente sobre a situação e sobre a confiança na sua capacidade de lidar com ela determinam a experiência de dor ${ }^{(5)}$. Desse modo, o julgamento do doente sobre a eficácia de vários tratamentos influi na efetividade das intervenções para o controle da queixa álgica ${ }^{(5)} \mathrm{e}$ fatores cognitivos podem exacerbar a dor e o sofrimento, contribuir para a incapacidade e influenciar na resposta à terapia ${ }^{(6-7)}$. Assim, identificar as atitudes, as crenças e os comportamentos pouco adaptativos e reorganizá-los é objetivo das intervenções de natureza cognitiva comportamental ${ }^{(6,8-9)}$.

$\mathrm{Na}$ área de dor crônica, a preocupação em conhecer e mensurar as atitudes dos doentes iniciou-se em torno de 1985. Para a avaliação das atitudes frente à dor foram elaborados alguns instrumentos: Pain Information and Beliefs Questionnaires - PIBQ ${ }^{(10)}$, Pain and Impairment Relationship Scale - PAIRS ${ }^{(11)}$, o instrumento denominado Pain Beliefs and Perceptions Inventory - $P B P I^{(12)}$ e o Survey of Pain Attitudes - SOPA ${ }^{(13-15)}$.

O SOPA é apontado como o melhor instrumento para a avaliação das atitudes dos doentes frente à dor crônica, com utilidade clínica e características psicométricas adequadas ${ }^{(8,16-17)}$. Foi organizado por em $1987^{(13)}$, e reformulado em 1991 e 1994 ${ }^{(13-15)}$. Em 1997 foi desenvolvida a versão breve do inventário Survey of Pain Attitudes (SOPA-B) ${ }^{(17)}$. O SOPA-B propõe-se a avaliar as atitudes de doentes com dor crônica não-oncológica. Consta de 30 itens, correspondentes a sete domínios de crenças e atitudes frente à dor: cura médica, controle, solicitude, incapacidade, medicação, emoção e dano físico. A validação desse instrumentos para a língua portuguesa foi o objetivo do presente estudo. A versão brasileira do SOPA-B será designada de Inventário de Atitudes frente à Dor Versão Breve (IAD-Breve).

Crenças são concepções muito antigas, culturalmente compartilhadas. São noções preexistentes sobre a percepção de nós mesmos, dos outros e do nosso ambiente. São consideradas verdades absolutas, exatamente o modo como as coisas $s \tilde{a} o^{(8,18-20)}$. Atitudes são disposições afetivas, relativamente estáveis, que implicam na tendência a responder ao alvo ou objeto (símbolo, frase, pessoa, instituição, idéia, crença, ideal, qualquer coisa existente para o indiví- duo), positiva ou negativamente, sendo culturalmente aprendidas e organizadas pela experiência. Envolvem orientação e intensidade em relação ao objeto-alvo. Orientação significa a aproximação ou rejeição de algo, ser favorável ou desfavorável, concordar ou discordar. Intensidade é a força com que se aprova ou desaprova o objeto. Pode-se aprovar ou desaprovar algo com intensidade diferentes, ou não se ter uma atitude frente a esse objeto. São disposições organizadas para a ação que se refletem no comportamento dos indivíduos e grupos ${ }^{(16,18-19,21-22)}$.

Cada um dos sete domínios do SOPA-B ${ }^{(13-15)}$ tem uma definição. $\mathrm{O}$ domínio de controle refere-se a quanto o doente acredita que pode controlar a sua dor (sua influência pessoal no controle da dor), e está representado pelas questões 1, 12, 17, 20 e 22 do IAD-breve (Anexo 1). Emoção refere-se a quanto o doente acredita que suas emoções influem na sua experiência dolorosa (relação entre emoção e intensidade da dor), e está representado pelos itens 6 , 10,15 e 25 . Incapacidade refere-se a quanto o doente acredita que está incapacitado pela dor (dor como incapacitante), e está representado pelas questões 23, 26 e 30 do inventário. Dano físico refere-se a quanto o doente acredita que a dor significa que está machucando a si mesmo e que deveria evitar exercícios (dor como indicativo de dano tecidual), e está representado pelos itens 11, 16, 19, 27 e 28 . Medicação refere-se a quanto o doente acredita que medicamentos são o melhor tratamento para dor crônica e está representado pelas questões 2,5 e 13 do instrumento. Solicitude refere-se a quanto o doente acredita que outros, especialmente os familiares, devem ser mais solícitos quando sente dor (solicitude de outros frente à pessoa com dor), e está representado pelos itens 3, 7, 9, 14 e 18. Cura médica refere-se a quanto o doente acredita na cura pela medicina para a sua dor (cura por meios médicos) e está representado pelas questões 4, 8, 21, 24 e 29.

O instrumento é auto-aplicado, e os respondentes indicam a concordância com cada uma das assertivas, em escala do tipo Likert de cinco pontos, que varia de 0 a $4(0=$ totalmente falso, $1=$ falso, $2=$ nem verdadeiro nem falso, $3=$ quase verdadeiro, $4=$ totalmente verdadeiro).

O escore de cada escala ou domínio é calculado pela soma dos pontos das repostas de cada item, dividido pelo número de itens respondidos. $\mathrm{O}$ escore final médio de cada escala poderá variar entre $0 \mathrm{e} 4$. Há itens invertidos $(4,8,11$, $23,24,26,27,28,29)$ que devem ter seus escores revertidos antes da soma. A reversão do escore faz-se subtraindo-se de 4 o escore escolhido pelo doente.

Não há pontos de corte, nem resposta certa ou errada; os escores dos sete domínios ou escalas não são somados. Há orientações de respostas mais desejáveis por serem consideradas, pelo autor do inventário, como hipoteticamente mais adaptativas. A orientação desejável dos es- 
cores para cada escala, depois do ajuste dos escores dos itens invertidos, é: controle $=4$; emoção=4; incapacidade $=0 ;$ dano físico $=0 ;$ medicação $=0 ;$ solicitude $=0$ e cura médica $=0$.

\section{MÉTODO}

Com a finalidade de alcançar equivalência conceitual entre a versão original e a brasileira foram realizados procedimentos de tradução, e a versão em português foi submetida a análise fatorial e medida de confiabilidade. A adaptação para o português do instrumento em estudo foi autorizada pelo autor da escala. $\mathrm{O}$ estudo foi autorizado pela instituição onde foi realizado e os participantes assinaram termo de consentimento livre e informado.

\section{Procedimentos de tradução}

A tradução para o português, reversão para o inglês (backtranslation) e comparação entre a versão original em inglês com a resultante da reversão foi feita por três diferentes tradutores que possuíam o inglês como língua materna. A cada etapa, a proposta resultante foi avaliada pelas autoras do estudo, que discutiram com os profissionais de língua inglesa as possíveis reformulações. Nesse sentido, as autoras atuaram como juízas, avaliando a equivalência do conteúdo e a equivalência semântica do inventário traduzido. Em entrevistas informais, não estruturadas, doentes do serviço onde seria testada a versão brasileira do IAD-Breve foram consultados sobre o significado, clareza e adequação na formulação dos itens. As sugestões ou manifestações de compreensão errônea sobre os conteúdos serviram de referência para o ajuste dos itens.

\section{Procedimentos de análise das propriedades psicométricas}

A validade de construto da versão brasileira foi estimada pela análise de propriedades psicométricas do IAD-Breve, aplicado em amostra de doentes brasileiros.

\section{Casuística}

Participaram do estudo 69 doentes com diagnóstico de dor crônica, em condições físicas e de compreensão adequadas para participar das entrevistas e que consentiram em fazer parte do estudo. Os dados foram coletados no segundo semestre de 1997 na Liga de Dor do Centro Acadêmico Osvaldo Cruz, da Faculdade de Medicina da Universidade de São Paulo e do Centro Acadêmico XXXI de Outubro, da Escola de Enfermagem da Universidade de São Paulo e Centro Multidisciplinar de Dor da Divisão de Clínica Neurológica do Hospital das Clínicas da Faculdade de Medicina da Universidade de São Paulo.
Dos 69 doentes, $71 \%$ eram mulheres; a idade média foi de $50,8( \pm 15,4)$ anos, e a mediana 50; a média de escolaridade foi $7,4( \pm 4,8)$ anos e, a mediana 8 ; a maior parte foi procedente das regiões sudeste $(56,5 \%)$ e nordeste $(27,5 \%) ; 46,4 \%$ eram aposentados ou estavam em licença médica, 39,1\% não possuíam atividade ocupacional remunerada e 14,5\% exerciam atividade remunerada; a dor era de etiologia muscular $(39,1 \%)$, neuropática $(20,3 \%)$, oncológica $(34,8 \%)$ e outras $(5,8 \%)$.

\section{$\underline{\text { Análise dos dados }}$}

Com o objetivo de verificar se as correlações entre os itens produziriam agrupamentos em fatores de forma semelhante aos do SOPA-B, utilizou-se o método de análise fatorial de componentes principais com rotação oblíqua ${ }^{(23)}$. A análise fatorial é importante para o estabelecimento da validade de construto de um instrumento para mensurar variáveis psicossociais. Trata-se de uma técnica estatística fundamentada no pressuposto de que uma série de variáveis observáveis pode ser explicada por uma série menor de variáveis hipotéticas, não observáveis, chamadas de fatores ${ }^{(24)}$.

A confiabilidade foi testada pelo $\alpha$ de Cronbach aplicado a cada uma das sete escalas do instrumento. Não se testou a confiabilidade sobre o total dos itens porque não se aplica um escore total ao conjunto das respostas do instrumento. A análise de consistência interna de um instrumento de coleta de dados é uma das técnicas para estimar a confiabilidade $^{(24)}$ e o coeficiente alfa $(\alpha)$ de Chronbach, utilizado no presente estudo, é o caso mais geral desse tipo de análise $\mathrm{e}^{(24)}$.

\section{RESULTADOS}

\section{Análise fatorial}

$\mathrm{Na}$ Tabela 1 apresentam-se as correlações (grandezas das cargas fatoriais) entre as assertivas (itens) e os fatores (domínios ou escalas). As assertivas correspondem à idéia de variável observável, comportamento empírico, e os fatores ao traço latente, neste caso, os domínios de atitude ${ }^{(24)}$.

A análise fatorial permitiu a solução com 7 fatores (domínios) (Tabela1) que produziram um percentual de explicação de $62,9 \%$. Os itens 19 e 21 não se agruparam nos domínios previstos para o SOPA ${ }^{(13-15)}$. Era esperado que o item 19 ('Alguma coisa está errada com o meu corpo que impede muito movimento ou exercício') compusesse o domínio Dano Físico e que o 21 ('Eu confio que a medicina pode curar a minha dor') ficasse em Cura Médica. O item 19 compôs Emoção e o 21 Medicação (Tabela 1). 
Tabela 1 - Matriz de correlações entre as assertivas do IAD - breve e os domínios segundo a análise fatorial (componentes principais) - São Paulo - 1998

\begin{tabular}{|c|c|c|c|c|c|c|c|}
\hline \multirow[t]{2}{*}{ Item } & \multicolumn{7}{|c|}{ Domínios ou escalas (Fatores) } \\
\hline & Controle & Incapacidade & Dano físico & Emoção & Medicação & Solicitude & Cura Médica \\
\hline 20 & 0,769 & $-0,350$ & $-0,187$ & 0,139 & 0,012 & $-0,009$ & $-0,035$ \\
\hline 1 & 0,716 & 0,073 & $-0,104$ & 0,096 & 0,041 & 0,289 & $-0,080$ \\
\hline 12 & 0,671 & $-0,278$ & $-0,068$ & 0,182 & $-0,066$ & 0,209 & $-0,027$ \\
\hline 17 & 0,646 & $-0,176$ & $-0,364$ & 0,335 & 0,124 & 0,221 & 0,113 \\
\hline 22 & 0,634 & $-0,052$ & $-0,286$ & 0,037 & $-0,134$ & $-0,017$ & $-0,074$ \\
\hline 26 & $-0,346$ & 0,770 & 0,238 & 0,208 & $-0,035$ & 0,113 & $-0,055$ \\
\hline 30 & $-0,007$ & 0,752 & 0,147 & $-0,022$ & 0,133 & 0,249 & 0,016 \\
\hline 23 & $-0,353$ & 0,373 & 0,217 & 0,315 & 0,359 & 0,065 & 0,156 \\
\hline 27 & $-0,356$ & 0,145 & 0,815 & $-0,126$ & 0,048 & $-0,002$ & 0,043 \\
\hline 11 & $-0,079$ & 0,110 & 0,766 & $-0,172$ & 0,059 & $-0,004$ & $-0,055$ \\
\hline 16 & $-0,117$ & 0,180 & 0,758 & 0,069 & 0,259 & $-0,203$ & $-0,149$ \\
\hline 28 & $-0,359$ & 0,320 & 0,701 & $-0,267$ & $-0,003$ & 0,099 & 0,004 \\
\hline 10 & 0,250 & 0,012 & $-0,106$ & 0,827 & 0,078 & 0,355 & $-0,116$ \\
\hline 15 & 0,230 & 0,043 & $-0,225$ & 0,812 & $-0,025$ & 0,338 & $-0,011$ \\
\hline 25 & 0,212 & $-0,002$ & $-0,311$ & 0,755 & $-0,080$ & 0,181 & $-0,177$ \\
\hline 6 & 0,382 & $-0,167$ & $-0,208$ & 0,713 & 0,059 & 0,387 & $-0,117$ \\
\hline 19 & $-0,272$ & 0,384 & 0,338 & 0,457 & 0,163 & $-0,113$ & 0,181 \\
\hline 5 & $-0,081$ & $-0,184$ & $-0,035$ & 0,032 & 0,818 & 0,087 & 0,044 \\
\hline 13 & 0,009 & 0,328 & 0,098 & $-0,246$ & 0,726 & 0,313 & $-0,001$ \\
\hline 2 & 0,002 & 0,292 & 0,339 & 0,217 & 0,555 & $-0,042$ & $-0,087$ \\
\hline 21 & 0,265 & $-0,132$ & 0,239 & $-0,017$ & 0,386 & 0,263 & 0,273 \\
\hline 3 & 0,157 & 0,135 & $-0,067$ & 0,234 & 0,034 & 0,863 & $-0,060$ \\
\hline 7 & $-0,066$ & 0,211 & 0,014 & 0,275 & 0,045 & 0,822 & 0,053 \\
\hline 18 & 0,294 & 0,154 & 0,245 & 0,175 & 0,173 & 0,729 & $-0,160$ \\
\hline 14 & 0,179 & 0,098 & $-0,050$ & 0,265 & 0,077 & 0,688 & $-0,020$ \\
\hline 9 & 0,080 & 0,037 & $-0,013$ & $-0,015$ & 0,252 & 0,667 & 0,015 \\
\hline 29 & $-0,312$ & $-0,192$ & $-0,056$ & 0,163 & 0,191 & $-0,125$ & 0,713 \\
\hline 8 & 0,039 & $-0,062$ & 0,091 & 0,061 & 0,119 & $-0,017$ & 0,710 \\
\hline 4 & $-0,101$ & 0,122 & $-0,105$ & $-0,244$ & $-0,203$ & $-0,005$ & 0,607 \\
\hline 24 & 0,171 & 0,156 & $-0,283$ & 0,285 & $-0,209$ & $-0,019$ & 0,600 \\
\hline
\end{tabular}

Nos procedimentos de redução do SOPA foi observado, na versão longa (57 itens) e na versão reduzida (30 itens), que o item 19, também, não compôs o domínio Dano Físico como esperado e sim o domínio Incapacidade ${ }^{(17)}$. A Tabela 2 permite comparar algumas características da análise fatorial do presente estudo com a do SOPA-B original $^{(17)}$ frente aos domínios previstos para o SOPA na versão longa ${ }^{(13)}$.
Os resultados dos testes de confiabilidade, descritos a seguir, foram utilizados para decidir sobre a melhor localização dos itens 19 e 21, considerando-se, também, os resultados da análise fatorial.

Tabela 2 - Comparações entre as soluções das análises fatoriais do IAD - breve na versão em português e o original de Tait e Chibnall 1997 - São Paulo - 1998

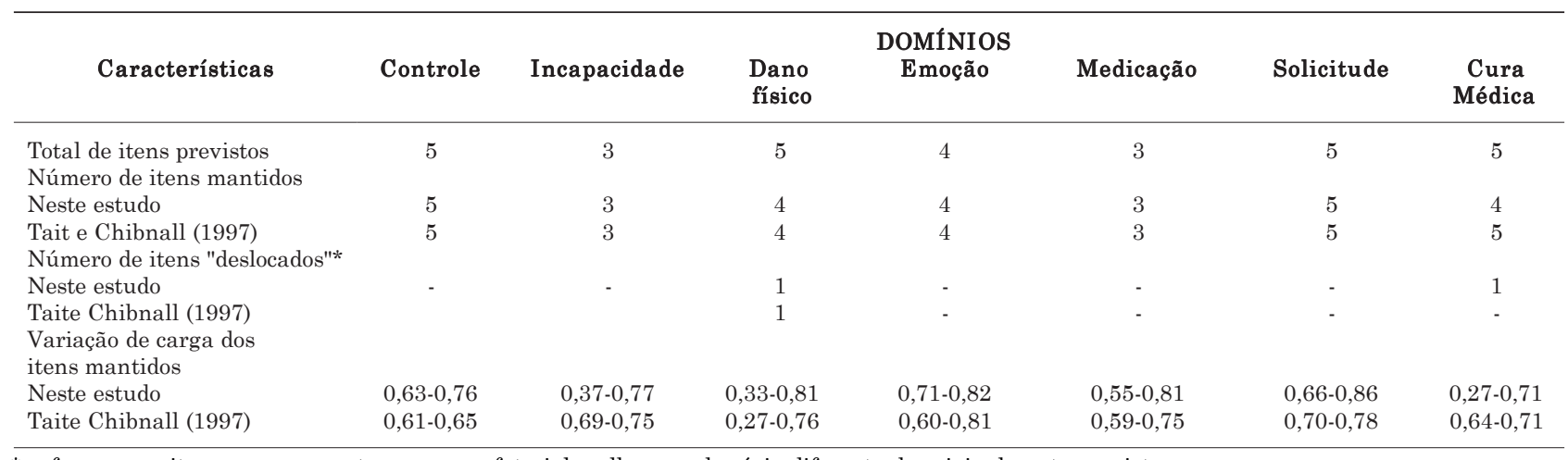

* refere-se aos itens que apresentaram carga fatorial melhor em domínio diferente do originalmente prevista 


\section{Análise de consistência interna}

A Tabela 3 apresenta os índices de confiabilidade obtidos em cada um dos sete fatores (domínios), a correlação de cada item com os outros do mesmo domínio e também o coeficiente

do domínio caso o item fosse excluído. Observa-se que os itens 19 e 21 estão localizados conforme o esperado e não conforme a análise fatorial (Tabela 1) do IAD-Breve.

Tabela 3 - Resultados da análise de consistência ( $\propto$ de Chronbach) do IAD - breve em língua portuguesa - São Paulo - 1998

\begin{tabular}{|c|c|c|c|}
\hline Domínio/Alfa & Ítens & Correlação com os outros ítens & Alfa se o item for excluído \\
\hline \multirow[t]{5}{*}{ Controle (Alfa: 0,7735) } & 1 & 0,4766 & 0,7546 \\
\hline & 12 & 0,5515 & 0,7314 \\
\hline & 17 & 0,5791 & 0,7201 \\
\hline & 20 & 0,6348 & 0,7017 \\
\hline & 22 & 0,4947 & 0,7487 \\
\hline \multirow[t]{4}{*}{ Emoção (Alfa: 0,8930) } & 6 & 0,7536 & 0,8659 \\
\hline & 10 & 0,7849 & 0,8541 \\
\hline & 15 & 0,8208 & 0,8405 \\
\hline & 25 & 0,6970 & 0,8861 \\
\hline \multirow[t]{3}{*}{ Incapacidade (Alfa: 0,6083) } & 23 & 0,3602 & 0,5994 \\
\hline & 26 & 0,6107 & 0,2056 \\
\hline & 30 & 0,3090 & 0,6487 \\
\hline \multirow[t]{5}{*}{ Dano físico (Alfa: 0,7693) } & 11 & 0,5882 & 0,7098 \\
\hline & 16 & 0,5936 & 0,7078 \\
\hline & 19 & 0,2609 & 0,8021 \\
\hline & 27 & 0,7086 & 0,6635 \\
\hline & 28 & 0,5491 & 0,7241 \\
\hline \multirow{3}{*}{ Medicação (Alfa: 0,5786) } & 2 & 0,2760 & 0,6580 \\
\hline & 5 & 0,4158 & 0,4333 \\
\hline & 13 & 0,4979 & 0,3350 \\
\hline \multirow{5}{*}{ Solicitude (Alfa: 0,8376) } & 3 & 0,7632 & 0,7678 \\
\hline & 7 & 0,7122 & 0,7850 \\
\hline & 9 & 0,5165 & 0,8380 \\
\hline & 14 & 0,5695 & 0,8234 \\
\hline & 18 & 0,6454 & 0,8032 \\
\hline \multirow{5}{*}{ Cura médica (Alfa: 0,5552) } & 4 & 0,3534 & 0,4806 \\
\hline & 8 & 0,3555 & 0,4809 \\
\hline & 21 & 0,1327 & 0,5809 \\
\hline & 24 & 0,3926 & 0,4509 \\
\hline & 29 & 0,3802 & 0,4757 \\
\hline
\end{tabular}

A análise fatorial (Tabela 1) mostrou que os itens 19 e 21 não se agruparam nos domínios previstos (Dano físico e Cura médica, respectivamente). Por isso foram feitas novas análises de consistência ( $\propto$ de Cronbach) transportando-se esses itens para os domínios em que tiveram maior carga na análise de componentes principais (Emoção e Medicação, respectivamente). $\mathrm{O}$ efeito dessas mudanças pode ser observado nas Tabelas 4 e 5.

Tabela 4 - Valores de $\propto$ do Item 19 nos domínios Dano físico e emoção - São Paulo - 1998

Item 19 - Alguma coisa está errada com o meu corpo que impede muito movimento ou exercício - originalmente previsto no domínio Dano físico mas com carga melhor no domínio Emoção

\begin{tabular}{|c|c|c|c|c|}
\hline \multirow{3}{*}{$\begin{array}{l}\text { Dano Físico } \\
\text { Item }\end{array}$} & \multicolumn{4}{|c|}{ Valores de $\propto$} \\
\hline & \multicolumn{2}{|c|}{ Com o item $19(0,7693)$} & \multicolumn{2}{|c|}{ Sem o item $19(0,8021)$} \\
\hline & Correlação com os outros itens & Alfa se o item for excluido & Correlação com os outros itens & Alfa se o item for excluido \\
\hline 11 & 0,5882 & 0,7098 & 0,6423 & 0,7392 \\
\hline 16 & 0,5936 & 0,7078 & 0,5330 & 0,7928 \\
\hline 27 & 0,7086 & 0,6635 & 0,7122 & 0,7043 \\
\hline 28 & 0,5491 & 0,7241 & 0,5821 & 0,7683 \\
\hline 19 & 0,2609 & 0,8021 & -- & -- \\
\hline \multirow[t]{2}{*}{ Emoção } & \multicolumn{4}{|c|}{ Valores de $\propto$} \\
\hline & \multicolumn{2}{|c|}{ Com o item $19(0,8925)$} & \multicolumn{2}{|c|}{ Sem o item $19(0,8930)$} \\
\hline Item & Correlação com os outros itens & Alfa se o item for excluido & Correlação com os outros itens & Alfa se o item for excluido \\
\hline 6 & 0,7027 & 0,7726 & 0,7536 & 0,8659 \\
\hline 10 & 0,7953 & 0,7424 & 0,7849 & 0,8541 \\
\hline 15 & 0,7978 & 0,7424 & 0,8208 & 0,8405 \\
\hline 25 & 0,6841 & 0,7786 & 0,6970 & 0,8861 \\
\hline 19 & 0,1608 & 0,8930 & -- & -- \\
\hline
\end{tabular}


Tabela 5 - Valores de $\propto$ do Item 21 nos domínios Cura médica e Medicação - São Paulo - 1998

Item 21 - Eu confio que a medicina pode curar a minha dor - originalmente previsto no domínio Cura médica mas com carga melhor no domínio Medicação

\begin{tabular}{|c|c|c|c|c|}
\hline \multirow{2}{*}{ Cura médica } & \multicolumn{4}{|c|}{ Valores de $\propto$} \\
\hline & \multicolumn{2}{|c|}{ Com o item $21(0,5552)$} & \multicolumn{2}{|c|}{ Sem o item $21(0,5809)$} \\
\hline Item & Correlação com os outros itens & Alfa se o item for excluido & Correlação com os outros itens & Alfa se o item for excluido \\
\hline 4 & 0,3534 & 0,4806 & 0,3775 & 0,5030 \\
\hline 8 & 0,3555 & 0,4809 & 0,3685 & 0,5115 \\
\hline 21 & 0,1327 & 0,5809 & -- & -- \\
\hline 24 & 0,3926 & 0,4509 & 0,3740 & 0,5076 \\
\hline 29 & 0,3802 & 0,4757 & 0,3773 & 0,5145 \\
\hline \multirow{2}{*}{ Medicação } & \multicolumn{4}{|c|}{ Valores de $\propto$} \\
\hline & \multicolumn{2}{|c|}{ Com o item $21(0,5826)$} & \multicolumn{2}{|c|}{ Sem o item $21(0,5786)$} \\
\hline Item & Correlação com os outros itens & Alfa se o item for excluido & Correlação com os outros itens & Alfa se o item for excluido \\
\hline 2 & 0,3062 & 0,5735 & 0,2760 & 0,6580 \\
\hline 5 & 0,4081 & 0,4747 & 0,4158 & 0,4333 \\
\hline 13 & 0,5149 & 0,3915 & 0,4979 & 0,3350 \\
\hline 21 & 0,2703 & 0,5786 & -- & -- \\
\hline
\end{tabular}

Foi decidido, com os argumentos apresentados na discussão a seguir, manter o item 19 no domínio Dano Físico e o item 21 no Cura Médica, tal como originalmente proposto $^{(13)}$.
A Tabela 6 permite observar o número de itens e os índices de confiabilidade de cada domínio obtidos com o IAD-Breve, neste estudo, e os obtidos na versão original $(\mathrm{SOPA}-\mathrm{B})^{(17)}$.

Tabela 6 - Índices de consistência interna das escalas do IAD - breve em língua inglesa e IAD - breve em língua portuguesa - São Paulo - 1998

\begin{tabular}{|c|c|c|c|c|}
\hline \multirow{2}{*}{ Domínios } & \multicolumn{2}{|c|}{ Tait e Chibnall (1997) } & \multicolumn{2}{|c|}{ Presente estudo } \\
\hline & $\propto$ & Número de ítens & $\propto$ & Número de ítens \\
\hline Solicitude & 0,83 & 5 & 0,83 & 5 \\
\hline Emoção & 0,80 & 4 & 0,89 & 4 \\
\hline Cura médica & 0,72 & 5 & 0,55 & 5 \\
\hline Controle & 0,70 & 5 & 0,77 & 5 \\
\hline Dano físico & 0,71 & 4 & 0,76 & 4 \\
\hline Incapacidade & 0,70 & 4 & 0,60 & 4 \\
\hline Medicação & 0,56 & 3 & 0,57 & 3 \\
\hline
\end{tabular}

\section{DISCUSSÃO}

Instrumentos de medidas submetidos a procedimentos de versão e adaptação lingüística podem ter a estrutura interna modificada ${ }^{(25)}$, daí a necessidade de avaliação das propriedades psicométricas. $\mathrm{O}$ instrumento que depois de traduzido mostra propriedades psicométricas semelhantes às do original pode ser considerado adaptado para o idioma alvo.

Neste estudo, a realização da análise fatorial teve como finalidade verificar se a estrutura de domínios (fatores), após a versão para o português, mantinha-se semelhante à original.

A análise fatorial do IAD-breve mostrou comportamento bastante satisfatório. A Tabela 2 mostra que o número de domínios, sete, observados no presente estudo, é igual ao do SOPA-B. As grandezas das cargas, na maioria dos itens, mostraram-se bastante próximas às obtidas com o SOPA-B ${ }^{(17)}$, e o percentual de explicação também é semelhante ao do original. Excetuando-se dois itens $(19,21)$, os demais se agruparam nos domínios teoricamente previstos (Tabela 1).

$\mathrm{O} \alpha$ de Chronbach verifica a congruência que cada item do instrumento tem com o restante dos itens do mesmo teste. O coeficiente pode variar de 0 a 1 , refletindo o grau de covariância dos itens entre si, considerando que a consistência interna do teste depende da natureza do traço que está 
sendo medido, do estágio de desenvolvimento do instrumento e do procedimento usado para estimar a confiabilidade ${ }^{(26)}$. Índices superiores a 0,50 são aceitos por alguns autores e outros recomendam valores acima de $0,70^{(27)}$.

No presente estudo, conforme pode-se notar na Tabela 3, os domínios controle, emoção, dano físico e solicitude tiveram a superior a 0,76. Nos domínios incapacidade, medicação e cura médica o a foi entre 0,55 e 0,60 , indicando que a confiabilidade nesses domínios foi moderada. É importante notar que o índice de confiabilidade tende a ser menor quando o número de itens é pequeno ${ }^{(28)}$, o que ocorreu nos domínios do instrumento em estudo, em que o número de itens variou de três a cinco.

Comparando os índices obtidos na versão brasileira aos obtidos com o SOPA-B original ${ }^{(17)}$, a Tabela 6 mostra que o domínio "Medicação" teve o mesmo índice nos dois estudos e que o domínio "Cura Médica", no presente estudo, apresentou índice de confiabilidade $(0,55)$ mais distante do obtido com a versão em inglês $(0,72)$. No presente estudo, a confiabilidade dos domínios foi considerada para decidir sobre o melhor posicionamento dos itens 19 e 21 , que se agruparam em fatores diferentes do esperado.

Para o item 19 estavam em questão os domínios "dano físico" e "emoção". Apesar de que o valor de $\alpha$ do domínio Dano Físico melhorou com a exclusão do item 19 (de 0,76 para 0,80 ), o que pode ser observado na Tabela 4 , a sua inclusão no domínio Emoção diminuiu o coeficiente de consistência nesse domínio (de 0,89 para 0,82 ). Na validação do SOPA-B original o valor de $\alpha$ no domínio Dano físico foi 0,71 . Esses argumentos são favoráveis à manutenção do item 19 em Dano Físico. No entanto, o item 19 apresentou baixos índices de correlação com os outros itens (Tabela 4), quando alocado no domínio Dano Físico $(0,26)$ ou no domínio Emoção $(0,16)$. Além disso, como pode-se observar na Tabela 1, a carga fatorial do item 19 no domínio Dano Físico foi 0,33 , valor muito próximo ao que se considera como pouco relevante (abaixo de 0,30$)^{(29)}$. É importante observar que no estudo com o SOPA-B original ${ }^{(17)}$ o item 19 não se localizou no domínio previsto (Dano Físico) mas sim no de Incapacidade. Esses argumentos são favoráveis à exclusão completa do item 19 do IAD-Breve. No entanto, sua permanência não interferiu na confiabilidade da escala, e este foi $o$ primeiro teste do IAD-Breve. Optou-se por manter o item 19 no domínio Dano Físico, conforme foi originalmente proposto, pois sua manutenção permitirá que ele seja testado em outros estudos e que se acumulem indicativos sobre sua importância no IAD-Breve.

O item 21 foi previsto no domínio Cura médica, mas teve carga maior no domínio Medicação (Tabela 1). A Tabela 5 mostra que a introdução desse item no domínio Cura Médica diminuiu o $\alpha$ de Crombach dessa escala (de 0,58 para 0,55). A exclusão do item 21 do domínio Medicação pouco modificou a consistência da escala (de 0,58 para 0,57 ). Na validação do SOPA-B original ${ }^{(17)}$, o valor de $\alpha$ para o domínio Cura médica foi 0,56 , bastante próximo ao observado no presente estudo quando o item 21 foi alocado no domínio Medicação. Quanto às cargas fatoriais, no presente estudo, o item 21 obteve 0,38 em Medicação e 0,27 em Cura Médica. Tais argumentos foram favoráveis à manutenção do item 21 no domínio Cura Médica. No entanto, considerando-se o limite $^{(29)}$ de 0,30 , esse item teve pouca relevância nos dois domínios. Decidimos manter o item 21 no domínio Medicação para que outros testes sejam realizados com o IADBreve e a adequação desse item seja novamente analisada.

\section{CONCLUSÃO}

Este estudo disponibiliza um instrumento para avaliação de crenças sobre dor crônica em língua portuguesa, o que permitirá a realização de testes empíricos dessa variável em população brasileira. A utilização do IAD-Breve na clínica facilita a avaliação do doente e o estabelecimento de intervenções.

No IAD-Breve foram observados 7 domínios, assim como no original em língua inglesa (SOPA-B). Os itens de cada um dos domínios também ficaram semelhantes ao original. Tais fatos indicam que há equivalência conceitual entre as duas versões, isto é, ambas medem os mesmos fenômenos. Outro indicativo de que houve adequada adaptação do instrumento para a língua portuguesa é que os índices de confiabilidade das escalas podem ser considerados bons ou moderados, e bastante semelhantes entre as duas versões.

Estudos sobre as propriedades psicométricas do IADBreve com amostras maiores permitirão melhor descrição da equivalência conceitual com a versão original. Recomenda-se que estimativas de confiabilidade sejam determinadas a cada vez que o teste for utilizado ${ }^{(30)}$, que cuidadosa observação sobre o comportamento dos itens 19 e 21 e que outros testes de validade e confiabilidade do instrumento sejam realizados. 


\section{REFERÊNCIAS}

(1) Merskey H, Bogduk N. Classification of chronic pain: descriptions of chronic pain syndromes and definitions of pain terms. $2^{\text {nd }}$ ed. Seattle: IASP; 1994.

(2) Pimenta CAM. Atitudes de doentes com dor crônica frente à dor [tese livre-docência]. São Paulo: Escola de Enfermagem da USP; 1999.

(3) Fordyce WE. Behavioral methods for chronic pain and illness Saint Louis: Mosby; 1976.

(4) Keefe FJ, Lefebvre JC. Behaviour therapy. In: Wall P, Melzack R, editors. Textbook of pain. $3^{\text {rd }}$ ed. Edinburgh: Churchill Livingstone; 1994. p. 1376-80.

(5) Turk DC, Meichenbaum D, Genest M. Pain and behavioral medicine: a cognitive behavioral perspective. New York: Guilford; 1983.

(6) Turk DC, Meichenbaum D. A cognitive-behavioral approach to pain management. In: Wall P, Melzack R, editors. Textbook of pain. Edinburgh: Churchill Livingstone; 1994. p. 1337-48.

(7) Pimenta CAM. Aspectos afetivos, culturais e terapêuticos relacionados à dor no câncer [tese]. São Paulo: Universidade de São Paulo; 1995.

(8) DeGood DE, Shutty MS. Assessment of pain beliefs, coping, and self-efficacy. In: Turk DC, Melzack R, editors. Handbook of pain assessment. New York: Guilford; 1992. p. 214-35.

(9) Bradley LAA. Cognitive behavioral therapy for chronic pain. In: Gatchel RJ, Turk DC, editors. Psychological approaches to pain management: a practitioner's handbook. New York: Guilford; 1996. p. 131-47.

(10) Schwartz DP, DeGood DE, Shutty MS. Direct assessment of beliefs and attitudes of chronic pain patients. Arch Phys Med Rehabil. 1985;66(12):806-9.

(11) Riley JF, Ahern DK, Follick MJ. Chronic pain and functional impairment: assessing beliefs about their relationship. Arch Phys Med Rehabil. 1988;69(8):579-82.

(12) Williams DA. An empirical assessment of pain beliefs. Pain. 1989;36(3):351-8.

(13) Jensen MP, Karoly P, Huger R. The development and preliminary validation of an instrument to assess patient's attitudes toward pain. J Psychosom Res. 1987;31(3): 393-400.

(14) Jensen MP. Control beliefs, coping efforts, and adjustment to chronic pain. J Consult Clin Psychol. 1991;59(3):431-8.

(15) Jensen MP, Turner JA, Romano JM, Lawler BK. Relationship of pain-specific beliefs to chronic pain adjustment. Pain. 1994;57(3):301-9.
(16) Strong J, Ashton R, Chant D. The measurement of attitudes towards and belief about pain. Pain. 1992;38(2):227-36.

(17) Tait RC, Chibnall JT. Development of a brief version of the Survey of Pain Attitudes. Pain. 1997;70(2-3):229-35.

(18) Chaplin JP. Dictionary of psychology. $2^{\text {nd }}$ ed. New York: Laurel; 1985.

(19) Anderson LW. Attitude measurement. In: Keeves SP, editor. Educational research methodology and measurement: an international handbook. Oxford: Pergamon; 1988. p. 421-8.

(20) Beck JS. Terapia cognitiva: teoria e prática. Porto Alegre: Artes Médicas; 1997.

(21) Ajzen I, Fishbein M. Attitude-behavior relations: a theoretical analysis and review of empirical research. Psychol Bull. 1977;84(5):888-918.

(22) Edwards AL. Techniques of attitude scale construction. New York: Appleton; 1957.

(23) Hair JF, Anderson RE, Tatham RL, Black WC. Multivariate data analysis. $5^{\text {th }}$ ed. New Jersey: Prentice Hall; 1998.

(24) Pasquali L. Psicometria: teoria e aplicações. Brasília: Universidade de Brasília.; 1997.

(25) Varricchio CG. Measurement issues co linguistic translations. In: Frank-Stromborg M, Olsen SJ, editors. Instrument for clinical health-care research. Boston: Jones and Bartlett; 1997. p. 54-63.

(26) Jacobson SF. Evaluating instruments for use in clinical nursing research. In: Frank-Stromborg M, Olsen SJ, editors. Instruments for clinical health-care research. Boston: Jones and Bartlett; 1997. p. 3-19.

(27) McDowell I, Newell C. The theoretical and technical foundations of health measurement. In: McDowell I, Newell C, editors. Measurin health: a guide to rating scales and questionnaires. $2^{\text {nd }}$ ed. New York: Oxford University; 1996. p. $10-46$.

(28) Nunnaly JC, Bernstein IH. Psychometric theory. $3^{\text {rd }}$ ed. New York: McGraw-Hill; 1994.

(29) Kline P. An easy guide to factor analysis. London: Routledge; 1994.

(30) Waltz CF, Strickland OL, Lenz ER. Reliability and validity of norm-referenced measures. In: Waltz CF, Strickland OL, Lenz ER, editors. Measurement in nursing research. Philadelphia: Davis; 1991. p. 161-94. 


\section{ANEXO I}

INVENTÁRIO DE ATITUDES FRENTE À DOR (IAD - BREVE)

\begin{tabular}{|c|c|c|c|c|c|c|}
\hline & & $\begin{array}{c}\text { totalmente } \\
\text { falso }\end{array}$ & $\begin{array}{l}\text { quase } \\
\text { falso }\end{array}$ & $\begin{array}{c}\text { nem } \\
\text { verdadeiro } \\
\text { nem falso }\end{array}$ & $\begin{array}{c}\text { quase } \\
\text { verdadeiro }\end{array}$ & $\begin{array}{l}\text { totalmente } \\
\text { verdadeiro }\end{array}$ \\
\hline 1. & Muitas vezes eu consigo influenciar a intensidade da dor que sinto. & 0 & 1 & 2 & 3 & 4 \\
\hline 2. & Provavelmente eu sempre terei que tomar medicamentos para dor. & 0 & 1 & 2 & 3 & 4 \\
\hline 3. & $\begin{array}{l}\text { Sempre que eu sinto dor eu quero que a minha família me trate } \\
\text { melhor. }\end{array}$ & 0 & 1 & 2 & 3 & 4 \\
\hline 4. & Eu não espero cura médica para a minha dor. & 0 & 1 & 2 & $\overline{3}$ & 4 \\
\hline 5. & O maior alívio da dor que eu tive foi com o uso de medicamentos. & 0 & 1 & 2 & 3 & 4 \\
\hline 6. & A ansiedade aumenta a minha dor. & 0 & 1. & 2 & 3 & 4 \\
\hline 7. & $\begin{array}{l}\text { Sempre que eu sinto dor as pessoas devem me tratar com cuidado e } \\
\text { preocupação. }\end{array}$ & 0 & 1 & 2 & 3 & 4 \\
\hline 8. & $\begin{array}{l}\text { Eu desisti de buscar a completa eliminação da minha dor através } \\
\text { do trabalho da medicina. }\end{array}$ & 0 & 1 & 2 & 3 & 4 \\
\hline 9. & $\begin{array}{l}\text { E responsabilidade daqueles que me amam ajudarem-me quando } \\
\text { eu sentir dor. }\end{array}$ & 0 & 1 & 2 & $\overline{3}$ & 4 \\
\hline 10. & O estresse na minha vida aumenta a minha dor. & 0 & 1 & 2 & 3 & 4 \\
\hline 11 . & Exercício e movimento são bons para o meu problema de dor. & 0 & 1 & 2 & 3 & 4 \\
\hline 12. & Concentrando-me ou relaxando-me consigo diminuir a minha dor. & 0 & 1 & 2 & 3 & 4 \\
\hline 13. & Remédio é um dos melhores tratamentos para dor crônica. & 0 & 1 & 2 & 3 & 4 \\
\hline 14. & $\begin{array}{l}\text { A minha família precisa aprender a cuidar melhor de mim quando } \\
\text { eu estiver com dor. }\end{array}$ & 0 & 1 & 2 & 3 & 4 \\
\hline 15. & A depressão aumenta a dor que sinto. & 0 & 1 & 2 & 3 & 4 \\
\hline 16. & $\begin{array}{l}\text { Se eu me exercitasse poderia piorar ainda mais o meu problema de } \\
\text { dor. }\end{array}$ & 0 & 1 & 2 & 3 & 4 \\
\hline 17. & $\begin{array}{l}\text { Eu acredito poder controlar a dor que sinto mudando meus } \\
\text { pensamentos. }\end{array}$ & 0 & 1 & 2 & 3 & 4 \\
\hline 18. & $\begin{array}{l}\text { Muitas vezes quando eu estou com dor eu preciso de mais carinho } \\
\text { do que estou recebendo agora. }\end{array}$ & 0 & 1 & 2 & 3 & 4 \\
\hline 19. & $\begin{array}{l}\text { Alguma coisa está errada com o meu corpo que impede muito } \\
\text { movimento ou exercício. }\end{array}$ & 0 & 1 & 2 & 3 & 4 \\
\hline 20. & Eu aprendi a controlar a minha dor. & 0 & 1 & 2 & 3 & 4 \\
\hline 21. & Eu confio que a medicina pode curar a minha dor. & 0 & 1 & 2 & 3 & 4 \\
\hline 22. & Eu sei com certeza que posso aprender a lidar com a minha dor. & 0 & 1 & 2 & 3 & 4 \\
\hline 23. & A minha dor não me impede de levar uma vida fisicamente ativa. & 0 & 1 & 2 & 3 & 4 \\
\hline 24. & A minha dor física não será curada. & 0 & 1 & 2 & 3 & 4 \\
\hline 25. & $\begin{array}{l}\text { Há uma forte ligação entre as minhas emoçōes e a intensidade da } \\
\text { minha dor. }\end{array}$ & 0 & 1 & 2 & 3 & 4 \\
\hline 26. & $\begin{array}{l}\text { Eu posso fazer quase tudo tão bem quanto eu podia antes de ter o } \\
\text { problema da dor. }\end{array}$ & 0 & 1 & 2 & 3 & 4 \\
\hline 27. & $\begin{array}{l}\text { Se eu não fizer exercícios regularmente o problema da minha dor } \\
\text { continuará a piorar. }\end{array}$ & 0 & 1 & 2 & 3 & 4 \\
\hline 28. & O exercício pode diminuir a intensidade da dor que eu sinto. & 0 & 1 & 2 & 3 & 4 \\
\hline 29 . & $\begin{array}{l}\text { Estou convencido de que não há procedimento médico que ajude a } \\
\text { minha dor. }\end{array}$ & 0 & 1 & 2 & 3 & 4 \\
\hline 30. & A dor que sinto impediria qualquer pessoa de levar uma vida ativa. & 0 & 1 & 2 & 3 & 4 \\
\hline
\end{tabular}

Protocolo para pontuação do IAD - breve com 30 itens

\begin{tabular}{|c|c|c|}
\hline Sub-escala & Itens* & Variação \\
\hline Solicitude & $\begin{array}{|lllll|}3 & 7 & 9 & 14 & 18 \\
\end{array}$ & $0-20$ \\
\hline Emoção & $\begin{array}{llll}6 & 10 & 15 & 25\end{array}$ & $0-16$ \\
\hline Cura & \begin{tabular}{|lllll}
$(4)$ & $(8)$ & 21 & $(24)$ & $(29)$ \\
\end{tabular} & $0-20$ \\
\hline Controle & $\begin{array}{lllll}1 & 12 & 17 & 20 & 22 \\
\end{array}$ & $0-20$ \\
\hline Dano físico & (11) $1619(27)$ & $0-16$ \\
\hline Incapacidade & (23) (26) 30 & $0-16$ \\
\hline Medicação & \begin{tabular}{|lll}
2 & 5 & 13 \\
\end{tabular} & $0-12$ \\
\hline
\end{tabular}

* Os itens entre parêntesis devem ter os escores revertidos 